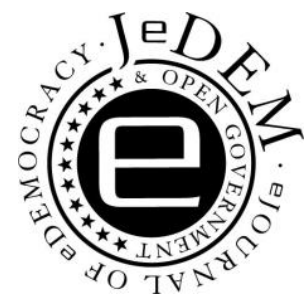

\title{
Mechanisms underpinning the usage of e- government services by businesses: A proposal based on previous empirical research
}

\section{Martha Correa-Ospina, ${ }^{1}$ Deepak Saxena, ${ }^{2}$ Beatriz Helena Díaz Pinzón ${ }^{3}$}

\author{
${ }^{1}$ ORCID Nr: 0000-0003-0865-4431 \\ Faculty of Engineering, Universidad Nacional de Colombia, mlcorreaos@unal.edu.co \\ ${ }^{2}$ ORCID Nr: 0000-0002-9331-3799 \\ Department of Management, Birla Institute of Technology and Science, deepak.saxena@pilani.bits- \\ pilani.ac.in \\ ${ }^{3}$ ORCID Nr: 0000-0002-9634-6297 \\ Faculty of Economic Sciences, Universidad Nacional de Colombia, bhdizap@unal.edu.co
}

\begin{abstract}
For many years, the public sector has been undergoing digital transformation. Information and communication technologies (ICT) have offered new ways of interaction between governments and their constituents. However, governments face different challenges to migrate users towards digital channels and electronic documents, which are believed to be more cost-efficient for all stakeholders. Despite a plethora of empirical research conducted towards the identification of factors that influence e-government services usage by businesses, there seems to be a lack of 'holistic' understanding in the absence of systematic literature reviews. This paper aims to contribute by hypothesizing a set of mechanisms based on a critical realist process of retroduction. We argue that the factors identified in previous research are a manifestation of mechanisms. Such mechanisms might explain businesses' usage of ICT when interacting with governments, whether in the context of incidental situations or regular administrative tasks (through online self-service applications) or regular exchange of information (through inter-organizational e-services).
\end{abstract}

Keywords: e-government, e-services, acceptance, adoption, mechanisms, businesses, critical realism

Acknowledgement: This work is part of a doctoral research at Universidad Nacional de Colombia, that is financially supported by the Ministry of Science, technology, and innovation through the scholarship program 757/2017. 


\section{Introduction}

In the information systems (IS) field, the adoption of technology is an area that has received wide attention. Various models have been proposed and validated within several contexts and towards the usage of different technologies. For many years, the public sector has been using information and communication technologies as part of digital transformation (Eggers \& Bellman, 2015; MacDonald, 2017; Mergel et al., 2018) to improve service delivery and its own internal efficiency, often under the e-government initiatives. Consequently, there is a growing body of academic literature on e-government and particularly, on the adoption of e-government services.

Since the success of any digital transformation initiative strongly relies on its usage, governments devise strategies to increase the usage of e-government services. Although governments may enforce the use of digital services by making it mandatory as it is the case of Denmark (Madsen \& Kræmmergaard, 2016), yet the users hold preferences and habits. Thus, migration towards electronic channels and electronic documents might be not as simple or straightforward. In addition, the supply-oriented approach in the design and implementation of electronic services seems to be still dominant, which is believed to affect the usage of public e-services (Scholta et al., 2020).

Among the users of e-government services, businesses are an important group. They have many legal obligations to fulfill as part of their operation. Apart from incidental or regular tasks that could be performed using online self-service applications, they also need to provide governments with large amounts of data. This information provision may not be necessarily in the interest of the business, yet may be mandated by the government due to its own interest (Arendsen \& van Engers, 2004).

Although previous literature reviews in the field of e-government have identified factors that influence e-government usage among different stakeholders, most are focused on G2C (government-to-citizen) services (Distel \& Ogonek, 2016; Hofmann et al., 2012; Nunes et al., 2017). Citizens as a group of users have received wide attention in terms of research focus. Factors influencing the usage of different types of services have been validated for informational and transactional services (Dwivedi et al., 2017), including the factors involved in citizen-participation (Lampoltshammer et al., 2019) and open government (Ruvalcaba-Gomez, 2019).

In contrast, empirical research on G2B (government-to-business) services is rather limited. Except for van den Boer et al. (2011) the research agenda towards channel choice in the G2B context, we did not find literature reviews that focus on technology-mediated encounters between business and government. Previous literature reviews on e-government have also noted the lack of studies in the business-to-government domain (Hofmann et al., 2012). This literature review contributes by filling this gap, with a particular focus on research in which the perspective of the businesses has been taken into account in empirical settings.

The main objective of this study is to gain a comprehensive understanding of the empirical research on businesses' usage of electronic services to interact with governments, by highlighting the factors that influence its usage. This paper also aims to go beyond the identification of a list of factors; using critical realist process of retroduction we postulate a list of hypothesized mechanisms 
(based of the empirical work reviewed) that help explain why businesses adopt e-government services.

This paper contributes to research by uncovering areas where research is needed. It also benefits practitioners as they can focus their attention on mechanisms underpinning a myriad of factors. Future work can consider the hypothesized mechanisms and examine them in empirical settings.

The remainder of this paper is organized as follows. Section 2 presents the literature review design; with classification and analysis of the papers reported in sections 3 and 4 . Section 5 presents and discusses the analysis towards the postulation of mechanisms (critical realist retroduction). Finally, section 6 presents the conclusions and future work directions.

\section{Review design}

The method for this literature review is divided into three parts: (1) literature search; (2) the classification framework; and (3) paper analysis.

\subsection{Literature Search}

Following Webster \& Watson (2002), our search for papers started with the Association of Business School (ABS) journal list for the areas of 'public sector' and 'information management' (of these journals, only the three-star and four-star journals were consulted), and the Senior Scholars' Basket of journals from the Association for Information Systems (AIS). Due to a focus on e-government, the search also includes the proceedings of the EGOV conference, and two open access journals that are specialized on e-government topics, Electronic Journal of E-Government (EJEG) and eJournal of eDemocracy \& Open Government (JeDEM). For database search to find additional papers, we used the Digital Government Reference Library (DGRL) version 15.5 and Scopus. And finally, we used Google Scholar to find papers that either referenced or were refered to by the papers selected in previous steps.

We purposely applied broad search terms to the selected sources to avoid the omission of pertinent work. Whilst we cannot claim to be exhaustive, results might be considered representative enough to draw conclusions of the research in the G2B domain regarding the adoption of egovernment services. We particularly review empirical work that involved businesses' perspective. The following basic search equation was used as search terms in titles, abstracts, and keywords: ("e-government" or "electronic government" or "d-government" or "digital government" or "online government" or "g2b" or "b2g") AND ("business" or "firm" or "enterprise" or "company" or "corporation" or "sme1" or "private sector") for the period 2000 - 2020.

This search resulted in the identification of 1955 papers. The papers were initially selected by scrutinizing the titles and abstracts. If the title did not give enough clarity about the content of the paper, the article was kept for the next stage of selection process. Based on this literature review's

${ }^{1}$ SME stands for Small and Medium-sized Enterprises 
main objective (of analyzing empirical research about the usage of e-government services from businesses' perspective), we ended with 434 papers pre-selected. After reading the abstracts 258 papers were omitted, this left 176 papers, of which 57 were discarded after reading the introduction. Once duplicates were removed, a total of 119 unique papers were considered for a full reading. Of these papers, the papers with unrelated topic were screened out. Finally, a total of 28 were included in the review. Backwards and forwards searches added ten to the final pool, bringing a total of 38 papers (see Figure 1).

Figure 1: Literature search flow chart

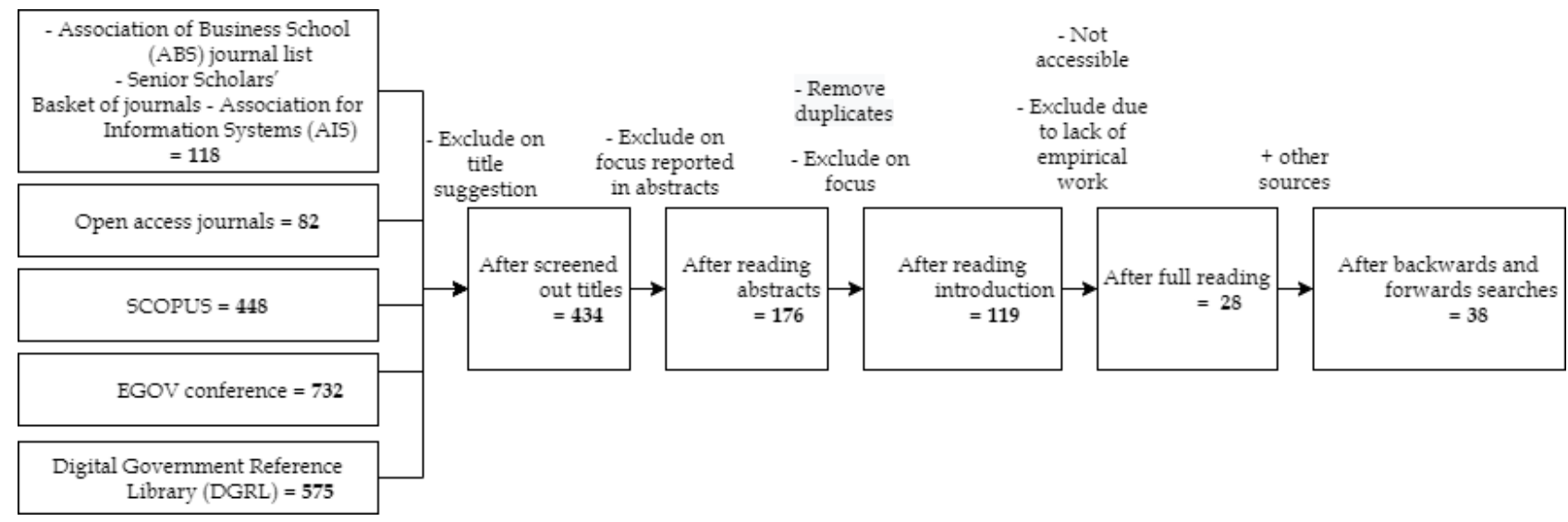

The selection criteria only considered papers which studied the usage of e-services by businesses to interact with governmental agencies and in which businesses side participated in. Thus, papers that only considered a supply perspective (even towards businesses sector), papers proposing or analyzing technological artifacts, proposing conceptual frameworks, or those only focusing on policymaking were excluded. Although some studies were found that used stakeholder's analysis, such studies were also left aside if the empirical work did not involve businesses. Appendix A presents the pool of papers included in this review.

\subsection{Classification framework}

For the coding of the papers selected, we define classification categories such as authors, year, journal. Moreover, since this literature review focuses on empirical research, we also identified the sample (business size) involved in the study and the country of data collection (see Appendix A). Additionally, research setting (Table 1) was identified based on the type of online services offered by the government.

Table 1: Classification of research settings

\begin{tabular}{|c|c|}
\hline $\begin{array}{c}\text { Research } \\
\text { setting }\end{array}$ & Description \\
\hline $\begin{array}{c}\text { Online Self- } \\
\text { service applica- } \\
\text { tion }\end{array}$ & $\begin{array}{c}\text { Self-service e-government services are web forms based, non-integrated } \\
\text { data exchange, usually accessed for occasional transactions as e.g. permits } \\
\text { and the registration of a new company (Arendsen et al., 2008). Informational }\end{array}$ \\
\hline
\end{tabular}




\begin{tabular}{|c|c|}
\hline $\begin{array}{c}\text { Inter- } \\
\text { organizational } \\
\text { systems }\end{array}$ & $\begin{array}{c}\text { services can be included in this category. Interaction is carried out by busi- } \\
\text { nesses to obtain information or complete a transaction by themselves. }\end{array}$ \\
$\begin{array}{c}\text { Such services perform electronic transaction processing based on mutual } \\
\text { data exchange as an integrated part of existing business management sys- } \\
\text { tems (e.g. tax filing, social security payments, e-invoicing, customs declara- } \\
\text { tions, and statistics) (Arendsen et al., 2008). This is similar to the use of Elec- } \\
\text { tronic Data Interchange (EDI) systems by businesses. }\end{array}$ \\
\hline
\end{tabular}

To analyze methodological aspects, we used the "research onion" (Saunders et al., 2019) as a base, and set the criteria to classify papers when those aspects were not explicitly mentioned in the paper.

Saunders et al. (2019) proposed the theoretical concept of "research onion" for research methodology construction. It provides a description of the main layers or stages for a methodology design. Research onion consists of six main layers, from research philosophy to techniques and procedures for data collection and analysis. We used this framework to classify our selected papers to get an overview of how the research in the field is being conducted. Tables 3 to 6 presents the classification criteria.

To classify papers on research philosophy we based our criteria on Saunders et al. (2019), and Saxena \& Mcdonagh (2017a). Table 2 notes the research philosophies and their description used in the review. Closely related to philosophy, we coded the papers based on their approach to theory development - deduction, induction, or abduction (Table 4).

Table 2: Research philosophy

\begin{tabular}{|c|c|}
\hline Research philosophy & Description \\
\hline Positivist & $\begin{array}{r}\text { Quantitative research that attempts to test theories (deductive } \\
\text { methods) to provide causal explanations and offer predictions. }\end{array}$ \\
\hline Critical realism & $\begin{array}{r}\text { Seeks for an explanation based on mechanisms. Research is } \\
\text { conducted following retroductive/abductive logic. }\end{array}$ \\
\hline Interpretativism & $\begin{array}{r}\text { Focuses on narratives, understandings, worldviews. Research is } \\
\text { conducted using inductive methods. }\end{array}$ \\
\hline Postmodernism & Focuses on the role of language and of power relations. \\
\hline Pragmatism & $\begin{array}{r}\text { The practical meaning of knowledge in particular contexts - } \\
\text { Problem-solving oriented (e.g., action research). }\end{array}$ \\
\hline
\end{tabular}


Table 3: Approaches to theory development

\begin{tabular}{|c|c|}
\hline $\begin{array}{c}\text { Approaches to theory } \\
\text { development }\end{array}$ & Description \\
\hline Deduction & $\begin{array}{r}\text { Studies moving from theory to data - Data collection aims to evalu- } \\
\text { ate propositions or hypotheses from an existing theory }\end{array}$ \\
\hline $\begin{array}{c}\text { Abduc- } \\
\text { tion/ Retroduction }\end{array}$ & Studies moving back and forth combining deduction and induction \\
\hline Induction & $\begin{array}{c}\text { Studies moving from data to theory - Data collection aims to explore } \\
\text { a phenomenon, identify themes and patterns to build theory. }\end{array}$ \\
\hline
\end{tabular}

The research strategy employed in the papers were classified (Table 4) according to the categories presented in the research onion, but some additional research strategies were included based on Madsen \& Kræmmergaard (2015). Although vignette studies use the surveys method as well, we decided to give it an independent category since vignette approach combines survey and experimental research (Table 5). Finally, the time horizon of the research was also identified (Table $6)$.

Table 4: Classification of research strategy

\begin{tabular}{|c|c|}
\hline Research strategy & Description \\
\hline Experiment & Papers using either laboratory or field experiments. \\
\hline Survey & Papers that gather data employing questionnaires. \\
\hline $\begin{array}{l}\text { Document analysis / } \\
\text { Archival research }\end{array}$ & $\begin{array}{c}\text { Papers that use internal or external documents as the primary data } \\
\text { source. }\end{array}$ \\
\hline Case study & $\begin{array}{l}\text { Papers presenting empirical investigation from a phenomenon in } \\
\text { its real settings where researcher has no control over events. }\end{array}$ \\
\hline Design science & Papers that build/develop systems and/or tools. \\
\hline Ethnography & $\begin{array}{l}\text { Papers which researcher collected data by immersing herself or } \\
\text { himself in the social world being researched, with a focus on thick } \\
\text { description. }\end{array}$ \\
\hline Action research & $\begin{array}{l}\text { Papers reporting research concerned with the resolution of organ- } \\
\text { izational issues as part of the research process. }\end{array}$ \\
\hline
\end{tabular}




\begin{tabular}{|c|c|}
\hline Grounded theory & $\begin{array}{l}\text { Papers emphasizing developing and building theory from the data } \\
\text { collected. }\end{array}$ \\
\hline Narrative inquiry & $\begin{array}{l}\text { Papers that collect data via interviews and focus on presenting the } \\
\text { stories. }\end{array}$ \\
\hline Focus group & Papers that collect data via focus groups. \\
\hline Vignette study & $\begin{array}{l}\text { Papers using short descriptions of a particular situation in which } \\
\text { the respondents need to empathize. }\end{array}$ \\
\hline
\end{tabular}

Table 5: Time Horizon

\begin{tabular}{|c|c|}
\hline Time Horizon & Description \\
\hline Cross-sectional & Studies at a particular instance of time, often using a wide num- \\
ber of respondents.
\end{tabular}

\subsection{Analysis of papers}

To synthesize the literature, a concept-centric analysis was conducted (Webster \& Watson, 2002). We identified the main themes of the research, main theoretical lenses, and variables/factors influencing e-government usage by businesses (presented in Section 4).

We also identified mechanisms that would explain why and under what circumstances businesses adopt and use e-government services based on a critical realist process of retroduction (Saxena, 2019). To do so, relevant factors (from the empirical research reviewed) were grouped according to their affinity and classified with regards to the context where they could be activated (inner or outer context of the organization).

\section{Classification of the papers}

In this section, we present the classification of the papers according to the classification framework discussed earlier. Regarding the size of business, figure 2 shows the distribution. While most studies do not consider a specific size or do not report a specific size of business (21 papers, labeled as "all sizes" in figure 2), 11 out of 38 explicitly focus on small and medium enterprises. In terms of the fieldwork setting, 21 different countries were identified, however, The Netherlands account for $26.3 \%$ of the studies (see Appendix A). Table 6 presents an overview of the research setting in terms of self-service applications and inter-organizational settings. 
Figure 2: Business size vs number of papers

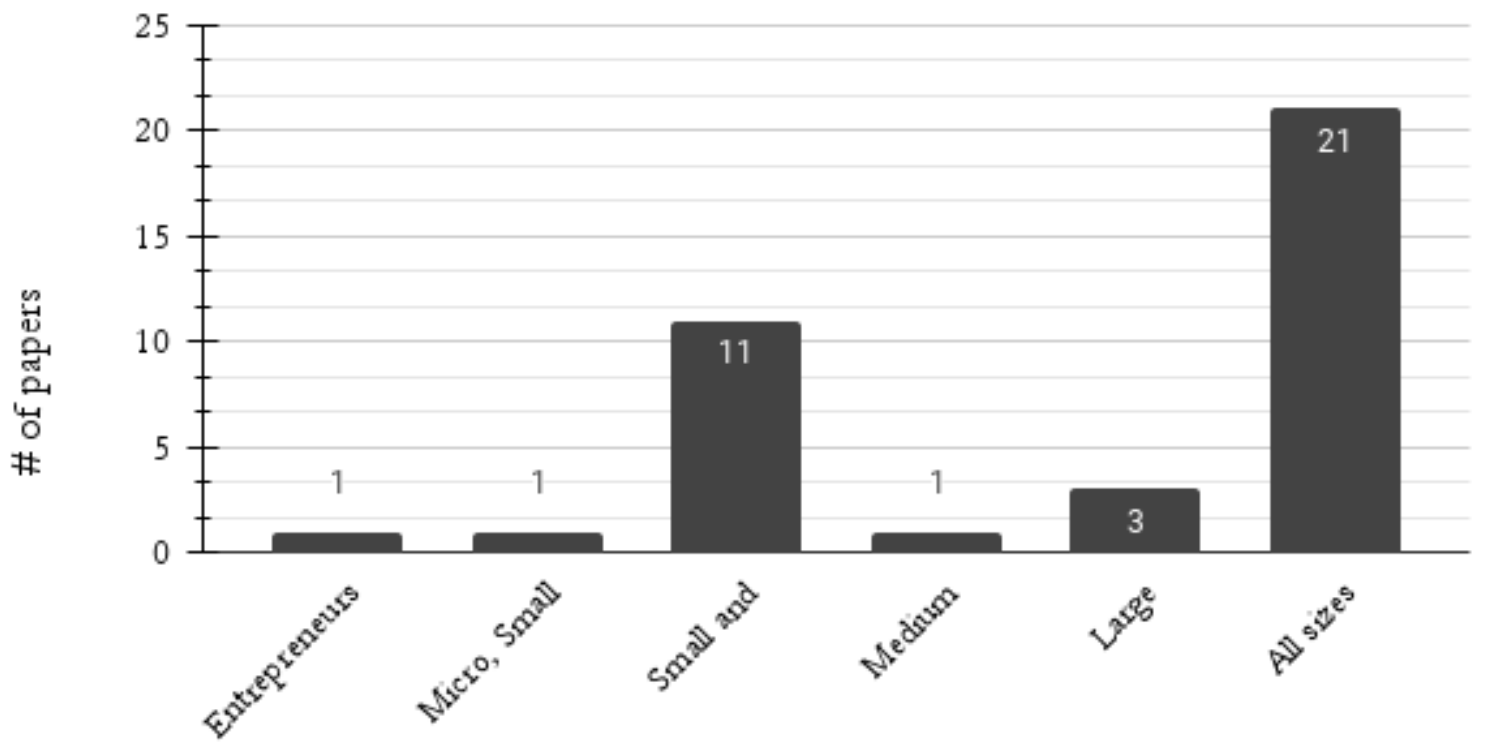

Sample (business size)

Table 6: Research settings

\begin{tabular}{|c|c|c|}
\hline & $\begin{array}{r}\text { \# of } \\
\text { papers }\end{array}$ & Papers \\
\hline $\begin{array}{l}\text { Self-service } \\
\text { applications }\end{array}$ & 24 & $\begin{array}{l}\text { (Alghamdi \& Beloff, 2016; Alomar \& de Visscher, 2017; Hung et } \\
\text { al., 2012; Jansen et al., 2010; Kindel et al., 2014; Lee et al., 2011; } \\
\text { Mbeche et al., 2017; Nguyen et al., 2020; Pinem et al., 2018; Ram- } \\
\text { mea \& Grobbelaar, 2017; Reddick \& Roy, 2013; Riyadh et al., 2019; } \\
\text { Santa et al., 2019; Seo et al., 2018; Shao et al., 2015; Soong et al., } \\
\text { 2020; Thi et al., 2014; Tung \& Rieck, 2005; Ulman et al., 2012; van } \\
\text { den Boer et al., 2012, 2016, 2017, 2014; Vejačka, 2018) }\end{array}$ \\
\hline $\begin{array}{c}\text { Inter- } \\
\text { organizational } \\
\text { systems }\end{array}$ & 14 & $\begin{array}{c}\text { (Arendsen et al., 2008, 2014, 2006; Arendsen \& Van De } \\
\text { Wijngaert, 2011; Bharosa et al., 2018; Gunasekaran et al., 2009; } \\
\text { Haag et al., 2013; Kassim \& Hussin, 2010; Lian, 2015; Mohd Nawi } \\
\text { et al., 2016; Naggi \& Agostini, 2011; Olaleye \& Sanusi, 2017; Sam- } \\
\text { basivan et al., 2010; Urciuoli et al., 2013) }\end{array}$ \\
\hline
\end{tabular}

In terms of research philosophy, most papers were found to be following a positivist research philosophy and a deductive approach towards theory development. Regarding methodological choice, 30 papers reported research relying on one quantitative method, 2 papers on quantitative and qualitative methods, and 4 papers on qualitative methods (see Table 7).

Table 7: Research philosophy and theory development approach

\begin{tabular}{|c|c|c|c|c|}
\hline $\begin{array}{c}\text { Research } \\
\text { philosophy }\end{array}$ & $\begin{array}{c}\text { Theory } \\
\text { development }\end{array}$ & $\begin{array}{c}\text { Methodo- } \\
\text { logical choice }\end{array}$ & $\begin{array}{c}\# \\
\text { of pa- }\end{array}$ & Papers \\
\hline
\end{tabular}




\begin{tabular}{|c|c|c|c|c|}
\hline & approach & & pers & \\
\hline \multirow[t]{2}{*}{ Positivist } & \multirow[t]{2}{*}{ Deductive } & $\begin{array}{l}\text { Mono- } \\
\text { method - Quan- } \\
\text { titative }\end{array}$ & 32 & $\begin{array}{l}\text { (Alghamdi \& Beloff, 2016; } \\
\text { Alomar \& de Visscher, 2017; Ar- } \\
\text { endsen et al., 2008, 2014, 2006; Ar- } \\
\text { endsen \& Van De Wijngaert, 2011; } \\
\text { Gunasekaran et al., 2009; Hung et } \\
\text { al., 2012; Jansen et al., 2010; Kindel } \\
\text { et al., 2014; Lee et al., 2011; Lian, } \\
\text { 2015; Mbeche et al., 2017; Nguyen et } \\
\text { al., 2020; Olaleye \& Sanusi, 2017; } \\
\text { Pinem et al., 2018; Reddick \& Roy, } \\
\text { 2013; Riyadh et al., 2019; Samba- } \\
\text { sivan et al., 2010; Santa et al., 2019; } \\
\text { Seo et al., 2018; Shao et al., 2015; } \\
\text { Soong et al., 2020; Thi et al., 2014; } \\
\text { Tung \& Rieck, 2005; Ulman et al., } \\
\text { 2012; Urciuoli et al., 2013; van den } \\
\text { Boer et al., 2012, 2016, 2017, 2014; } \\
\text { Vejačka, 2018) }\end{array}$ \\
\hline & & $\begin{array}{c}\text { Mixed- } \\
\text { method simple }\end{array}$ & 2 & $\begin{array}{l}\text { (Bharosa et al., 2018; Haag et al., } \\
\text { 2013) }\end{array}$ \\
\hline $\begin{array}{l}\text { Interpreta- } \\
\text { tivism }\end{array}$ & Inductive & $\begin{array}{l}\text { Mono- } \\
\text { method - Quali- } \\
\text { tative }\end{array}$ & 4 & $\begin{array}{l}\text { (Kassim \& Hussin, 2010; Mohd } \\
\text { Nawi et al., 2016; Naggi \& Agostini, } \\
\text { 2011; Rammea \& Grobbelaar, 2017) }\end{array}$ \\
\hline
\end{tabular}

Table 8 presents the time horizon of the research and the strategy among the papers. Survey (30 papers), vignette studies ( 3 papers), and case study (5 papers) were the three strategies identified, meaning, almost all studies are cross-sectional in nature, but one conducted survey at three different moments in time. On the other hand, interestingly, all the vignette studies were conducted in The Netherlands and towards source and channel choice within the businesses' information seeking process.

Table 8: Time horizon and research strategy

\begin{tabular}{|c|c|c|c|}
\hline $\begin{array}{r}\text { Time } \\
\text { horizon }\end{array}$ & Strategy & $\begin{array}{r}\text { \# of } \\
\text { papers }\end{array}$ & Papers \\
\hline \multirow[t]{3}{*}{$\begin{array}{l}\text { Cross- } \\
\text { sectional }\end{array}$} & Survey & 29 & $\begin{array}{l}\text { (Alghamdi \& Beloff, 2016; Alomar \& de Visscher, } \\
\text { 2017; Arendsen et al., 2008, 2014, 2006; Gunasekaran et } \\
\text { al., 2009; Haag et al., 2013; Hung et al., 2012; Lee et al., } \\
\text { 2011; Lian, 2015; Mbeche et al., 2017; Nguyen et al., } \\
\text { 2020; Olaleye \& Sanusi, 2017; Reddick \& Roy, 2013; Ri- } \\
\text { yadh et al., 2019; Sambasivan et al., 2010; Santa et al., } \\
\text { 2019; Seo et al., 2018; Shao et al., 2015; Soong et al., } \\
\text { 2020; Thi et al., 2014; Tung \& Rieck, 2005; Urciuoli et } \\
\text { al., 2013; van den Boer et al., 2012, 2016, 2017; Vejačka, } \\
\text { 2018) }\end{array}$ \\
\hline & $\begin{array}{l}\text { Vignette } \\
\text { Study }\end{array}$ & 3 & $\begin{array}{l}\text { (Arendsen \& Van De Wijngaert, 2011; Jansen et al., } \\
\text { 2010; van den Boer et al., 2014) }\end{array}$ \\
\hline & Case & 5 & (Bharosa et al., 2018; Kassim \& Hussin, 2010; Mohd \\
\hline
\end{tabular}




\begin{tabular}{|c|c|c|c|}
\hline & study & & $\begin{array}{c}\text { Nawi et al., 2016; Naggi \& Agostini, 2011; Rammea \& } \\
\text { Grobbelaar, 2017) }\end{array}$ \\
\hline $\begin{array}{c}\text { Longitu- } \\
\text { dinal }\end{array}$ & Survey & 1 & (Ulman et al., 2012) \\
\hline
\end{tabular}

\section{Concept-centric analysis of the literature review}

In this section we present a concept-centric analysis (Webster \& Watson, 2002) of the pool of papers. Table 9 presents the result of the themes identification across the papers and table 11 the main theoretical lenses underlying the studies.

\begin{tabular}{|c|c|c|}
\hline $\begin{array}{l}\text { Table 9: Main themes of } \\
\text { researchTheme }\end{array}$ & $\begin{array}{r}\text { \# of } \\
\text { papers }\end{array}$ & Papers \\
\hline $\begin{array}{c}\text { General e-services } \\
\text { (or more than one ser- } \\
\text { vice) }\end{array}$ & 12 & $\begin{array}{l}\text { (Alghamdi \& Beloff, 2016; Kindel et al., 2014; Mbeche et al., } \\
\text { 2017; Nguyen et al., 2020; Rammea \& Grobbelaar, 2017; Red- } \\
\text { dick \& Roy, 2013; Riyadh et al., 2019; Santa et al., 2019; Thi et } \\
\text { al., 2014; Tung \& Rieck, 2005; Ulman et al., 2012; Vejačka, } \\
\text { 2018) }\end{array}$ \\
\hline $\begin{array}{l}\text { Informational e- } \\
\text { services }\end{array}$ & 5 & $\begin{array}{l}\text { (Jansen et al., 2010; van den Boer et al., 2012, 2016, 2017, } \\
\text { 2014) }\end{array}$ \\
\hline $\begin{array}{c}\text { E-invoicing } \\
\text { (Arendsen \& Van De Wijngaert, 2011; Haag et al., 2013; } \\
\text { Lian, 2015; Naggi \& Agostini, 2011; Olaleye \& Sanusi, 2017) }\end{array}$ \\
\hline $\begin{array}{l}\text { E-procurement } \\
\text { System }\end{array}$ & 8 & $\begin{array}{l}\text { Hung et al., 2012; Kassim \& Hussin, 2010; Mohd Nawi et al., } \\
\text { 2016; Sambasivan et al., 2010; Seo et al., 2018; Soong et al., } \\
\text { 2020) }\end{array}$ \\
\hline $\begin{array}{l}\text { E-tax filing } \\
\text { High impact gov- } \\
\text { ernmental e-services } \\
\text { (reduction of adminis- } \\
\text { trative burden) }\end{array}$ & 3 & $\begin{array}{l}\text { (Lee et al., 2011; Shao et al., 2015) } \\
\text { (Arendsen et al., 2008, 2014, 2006) }\end{array}$ \\
\hline $\begin{array}{l}\text { E-customs } \\
\text { E-services for re- }\end{array}$ & 2 & $\begin{array}{l}\text { (Urciuoli et al., 2013) } \\
\text { (Bharosa et al., 2018; Pinem et al., 2018) }\end{array}$ \\
\hline
\end{tabular}

Regarding the theoretical lens, we found that almost all studies primarily draw from the theories employed in the IS field (Table 10). Although some papers use a main theory, model, or framework supporting their research models, most of the research is eclectic in approach.

Table 10: Main theoretical lense employed.

\begin{tabular}{|c|c|c|}
\hline $\begin{array}{c}\text { Theoretical } \\
\text { lenses }\end{array}$ & Description* & $\begin{array}{l}\text { Paper that adapts the } \\
\text { theory/framework }\end{array}$ \\
\hline
\end{tabular}




\begin{tabular}{|c|c|c|}
\hline $\begin{array}{l}\text { Theory of } \\
\text { Planned Behav- } \\
\text { ior (TPB) }\end{array}$ & $\begin{array}{l}\text { TPB is rooted in the social psychology } \\
\text { field, but it is also used to explain acceptance } \\
\text { towards a given technology. The three main } \\
\text { constructs in TPB are attitude, subjective } \\
\text { norm, and perceived behavioral control. }\end{array}$ & (Hung et al., 2012) \\
\hline $\begin{array}{l}\text { Channel } \\
\text { choice theories / } \\
\text { Information } \\
\text { seeking theories }\end{array}$ & $\begin{array}{l}\text { Theories of channel choice (e.g., Media } \\
\text { Richness Theory, the Social Influence Model, } \\
\text { and the Channel Expansion Theory). } \\
\text { Theories of source choices (e.g., Byström } \\
\text { and Järvelin's model of information seeking, } \\
\text { Leckie's model of information seeking). }\end{array}$ & $\begin{array}{l}\text { (Tung \& Rieck, 2005; van } \\
\text { den Boer et al., 2012, 2016, } \\
\text { 2017, 2014) }\end{array}$ \\
\hline $\begin{array}{l}\text { Chwelos, } \\
\text { Benbasat, and } \\
\text { Dexter's EDI } \\
\text { adoption model }\end{array}$ & $\begin{array}{l}\text { Model focusing on the adoption of inter- } \\
\text { organizational systems. It postulates that per- } \\
\text { ceived benefits, readiness, and external pres- } \\
\text { sure have a positive relationship with the } \\
\text { adoption decision (Chwelos et al., 2001). }\end{array}$ & $\begin{array}{l}\text { (Arendsen et al., 2008; } \\
\text { Tung \& Rieck, 2005) }\end{array}$ \\
\hline $\begin{array}{l}\text { Iacovou, Ben- } \\
\text { basat, and Dex- } \\
\text { ter's EDI adop- } \\
\text { tion and impact } \\
\text { model }\end{array}$ & $\begin{array}{l}\text { Model focusing on the adoption of inter- } \\
\text { organizational systems by small firms. It } \\
\text { states that three factors: organizational readi- } \\
\text { ness, external pressures, and perceived bene- } \\
\text { fits influence EDI adoption. }\end{array}$ & (Tung \& Rieck, 2005) \\
\hline $\begin{array}{c}\text { DeLone and } \\
\text { McLean's model }\end{array}$ & $\begin{array}{l}\text { This model was proposed by DeLone \& } \\
\text { McLean }(1992,2003) \text { to measure the infor- } \\
\text { mation system success. It takes into account } \\
\text { the information, system, and service quality, } \\
\text { user satisfaction and current usage and the } \\
\text { net of benefits. }\end{array}$ & (Sambasivan et al., 2010) \\
\hline $\begin{array}{l}\text { Expectation } \\
\text { confirmation } \\
\text { theory }\end{array}$ & $\begin{array}{l}\text { Expectations-confirmation theory posits } \\
\text { that expectations and perceived performance } \\
\text { lead to satisfaction. The four main constructs } \\
\text { are expectations, performance, disconfirma- } \\
\text { tion, and satisfaction. }\end{array}$ & (Pinem et al., 2018) \\
\hline $\begin{array}{l}\text { Technology } \\
\text { Acceptance } \\
\text { Model (TAM) }\end{array}$ & $\begin{array}{l}\text { It is an adaptation of the Theory of Rea- } \\
\text { soned Action (TRA) to the field of IS. It posits } \\
\text { that perceived usefulness and perceived ease } \\
\text { of use determine intention to use a system. }\end{array}$ & $\begin{array}{l}\text { (Alomar \& de Visscher, } \\
\text { 2017; Soong et al., 2020; } \\
\text { Vejačka, 2018) }\end{array}$ \\
\hline $\begin{array}{l}\text { Technology- } \\
\text { organization- } \\
\text { environment } \\
\text { (TOE) frame- } \\
\quad \text { work } \\
\end{array}$ & $\begin{array}{l}\text { It identifies three aspects that influence the } \\
\text { process by which an organization adopts and } \\
\text { implements a technological innovation: tech- } \\
\text { nological, organizational, and environmental } \\
\text { context. }\end{array}$ & $\begin{array}{l}\text { (Alomar \& de Visscher, } \\
\text { 2017; Arendsen et al., 2014, } \\
\text { 2006; Shao et al., 2015; Thi et } \\
\text { al., 2014) }\end{array}$ \\
\hline $\begin{array}{l}\text { E- } \\
\text { Government } \\
\text { Adoption and } \\
\text { Utilization Mod- } \\
\text { el (EGAUM) }\end{array}$ & $\begin{array}{l}\text { EGAUM was developed based on the lit- } \\
\text { erature on e-government adoption, and theo- } \\
\text { ries used to analyze acceptance and usage of } \\
\text { technologies. }\end{array}$ & (Alghamdi \& Beloff, 2016) \\
\hline $\begin{array}{l}\text { Unified Theo- } \\
\text { ry of Acceptance } \\
\text { and Use of }\end{array}$ & $\begin{array}{l}\text { These models aim to explain technology } \\
\text { acceptance and they are based on other tech- } \\
\text { nology acceptance theories. UTAUT } 2 \text { is par- }\end{array}$ & $\begin{array}{l}\text { (Lian, 2015; Nguyen et al., } \\
\text { 2020; Olaleye \& Sanusi, 2017; } \\
\text { Soong et al., 2020) }\end{array}$ \\
\hline
\end{tabular}




\begin{tabular}{|c|c|c|}
\hline $\begin{array}{l}\text { Technology / } 2 \\
\text { (UTAUT / 2) }\end{array}$ & ticularly adapted to the customer context. & \\
\hline $\begin{array}{l}\text { Rogers' Inno- } \\
\text { vation Diffusion } \\
\text { Theory (DOI) }\end{array}$ & $\begin{array}{l}\text { It states that the primary determinants of } \\
\text { innovation diffusion are innovation charac- } \\
\text { teristics: observability, trialability, complexi- } \\
\text { ty, relative advantage, and compatibility } \\
\text { (Rogers, 1995). }\end{array}$ & $\begin{array}{r}\text { (Alomar \& de Visscher, } \\
\text { 2017; Tung \& Rieck, 2005) }\end{array}$ \\
\hline $\begin{array}{l}\text { Human- } \\
\text { Organization- } \\
\text { Technology } \\
\text { (HOT) Fit } \\
\text { framework } \\
\text { which }\end{array}$ & $\begin{array}{l}\text { Socio-technical model that considers e- } \\
\text { government systems from a human, organi- } \\
\text { zational and technology perspective. }\end{array}$ & $\begin{array}{c}\text { (Rammea \& Grobbelaar, } \\
\text { 2017) }\end{array}$ \\
\hline $\begin{array}{l}\text { Various theo- } \\
\text { retical perspec- } \\
\text { tives }\end{array}$ & $\begin{array}{l}\text { Various models or literature on factors are } \\
\text { considered. }\end{array}$ & $\begin{array}{l}\text { (Arendsen et al., 2014; } \\
\text { Arendsen \& Van De Wijn- } \\
\text { gaert, 2011; Bharosa et al., } \\
\text { 2018; Gunasekaran et al., } \\
\text { 2009; Haag et al., 2013; Jan- } \\
\text { sen et al., 2010; Kindel et al., } \\
\text { 2014; Lee et al., 2011; Mbeche } \\
\text { et al., 2017; Reddick \& Roy, } \\
\text { 2013; Riyadh et al., 2019; } \\
\text { Santa et al., 2019; Seo et al., } \\
\text { 2018; Ulman et al., 2012; Ur- } \\
\text { ciuoli et al., 2013) }\end{array}$ \\
\hline $\begin{array}{l}\text { No specific } \\
\text { theory }\end{array}$ & & $\begin{array}{l}\text { (Kassim \& Hussin, 2010; } \\
\text { Mohd Nawi et al., 2016; } \\
\text { Naggi \& Agostini, 2011) } \\
\end{array}$ \\
\hline
\end{tabular}

*Source of some descriptions was https://is.theorizeit.org/wiki/Main_Page

Since the aim of this literature review is to gain a comprehensive understanding of businesses' usage of electronic services to interact with governments, Table 11 presents the main findings (based on the empirical works reviewed) on the factors influencing the usage of electronic services. Because most studies apply variance models to test the impact of some factors in a dependent variable, findings are grouped based on the dependent variable analyzed (intention to use, satisfaction, channel choice, etc.). The plus $(+)$ sign beside the factor indicates that the concerned factor supports the dependent variable. Similarly, the minus (-) sign indicates that the concerned factor acts as an obstacle for the dependent variable. Regarding channel choice studies, we highlight those factors influencing e-government services selection.

Table 11: Main factors influencing the usage of e-government services by businesses.

\begin{tabular}{|c|c|c|}
\hline $\begin{array}{c}\text { Depend- } \\
\text { ent variable }\end{array}$ & $\begin{array}{c}\text { Factors influencing depend- } \\
\text { ent variable }\end{array}$ & Paper(s) \\
\hline $\begin{array}{c}\text { Intention } \\
\text { to use }\end{array}$ & Perceived benefits (+) & $\begin{array}{c}\text { (Alghamdi \& Beloff, 2016; Nguyen et } \\
\text { al., 2020; Tung \& Rieck, 2005) }\end{array}$ \\
\hline
\end{tabular}




\begin{tabular}{|c|c|}
\hline External pressure $(+)$ & (Tung \& Rieck, 2005) \\
\hline Social influence $(+)$ & $\begin{array}{l}\text { (Arendsen \& Van De Wijngaert, 2011; } \\
\text { Lian, 2015; Olaleye \& Sanusi, 2017; Soong } \\
\text { et al., 2020; Tung \& Rieck, 2005) }\end{array}$ \\
\hline $\begin{array}{l}\text { Organizational IT experience } \\
(+)\end{array}$ & $\begin{array}{l}\text { (Arendsen et al., 2006; Arendsen \& } \\
\text { Van De Wijngaert, 2011) }\end{array}$ \\
\hline Operational performance $(+)$ & $\begin{array}{c}\text { (Arendsen et al., 2006; Gunasekaran et } \\
\text { al., 2009) }\end{array}$ \\
\hline $\begin{array}{c}\text { Firm's size / Organizational } \\
\text { size / Demographic characteris- } \\
\text { tics }(+/-)\end{array}$ & $\begin{array}{l}\text { (Alomar \& de Visscher, 2017; Ar- } \\
\text { endsen et al., 2006; Arendsen \& Van De } \\
\text { Wijngaert, 2011; Nguyen et al., 2020) }\end{array}$ \\
\hline Organizational readiness $(+)$ & $\begin{array}{c}\text { (Alomar \& de Visscher, 2017; Ar- } \\
\text { endsen et al., 2008; Naggi \& Agostini, } \\
\text { 2011) }\end{array}$ \\
\hline Perceived usefulness $(+)$ & $\begin{array}{l}\text { (Hung et al., 2012; Mbeche et al., 2017; } \\
\text { Mohd Nawi et al., 2016; Riyadh et al., } \\
\text { 2019; Sambasivan et al., 2010; Vejačka, } \\
\text { 2018) }\end{array}$ \\
\hline Fear of change $(-)$ & (Gunasekaran et al., 2009) \\
\hline Facilitating conditions $(+)$ & $\begin{array}{l}\text { (Hung et al., 2012; Nguyen et al., 2020; } \\
\text { Sambasivan et al., 2010) }\end{array}$ \\
\hline Ease of use $(+)$ & $\begin{array}{l}\text { (Mbeche et al., 2017; Sambasivan et al., } \\
\text { 2010; Urciuoli et al., 2013) }\end{array}$ \\
\hline $\begin{array}{l}\text { Web design (service quality) } \\
(+)\end{array}$ & $\begin{array}{l}\text { (Mbeche et al., 2017; Nguyen et al., } \\
\text { 2020; Sambasivan et al., 2010) }\end{array}$ \\
\hline $\begin{array}{l}\text { Assurance of service (by ser- } \\
\text { vice providers) }(+)\end{array}$ & $\begin{array}{c}\text { (Naggi \& Agostini, 2011; Sambasivan } \\
\text { et al., 2010) }\end{array}$ \\
\hline $\begin{array}{l}\text { Responsiveness of service pro- } \\
\text { viders }(+)\end{array}$ & $\begin{array}{l}\text { (Gunasekaran et al., 2009; Mohd Nawi } \\
\text { et al., 2016; Sambasivan et al., 2010) }\end{array}$ \\
\hline $\begin{array}{l}\text { Business opportunities / com- } \\
\text { petitiveness }(+)\end{array}$ & $\begin{array}{l}\text { (Kassim \& Hussin, 2010; Mbeche et al., } \\
\text { 2017) }\end{array}$ \\
\hline $\begin{array}{l}\text { Perception of high-quality of- } \\
\text { fline service provision }(+)\end{array}$ & (Lee et al., 2011) \\
\hline $\begin{array}{l}\text { Attitude towards change / IT } \\
\qquad(+)\end{array}$ & $\begin{array}{l}\text { (Alomar \& de Visscher, 2017; Ar- } \\
\text { endsen et al., 2014; Arendsen \& Van De } \\
\text { Wijngaert, 2011) }\end{array}$ \\
\hline Interpersonal influence $(+)$ & $\begin{array}{c}\text { (Hung et al., 2012; Olaleye \& Sanusi, } \\
\text { 2017) }\end{array}$ \\
\hline Perceived risk (-) & (Hung et al., 2012; Lian, 2015) \\
\hline External influence $(+)$ & (Hung et al., 2012) \\
\hline Self-efficacy $(+/-)$ & $\begin{array}{c}\text { (Hung et al., 2012; Kassim \& Hussin, } \\
\text { 2010) }\end{array}$ \\
\hline $\begin{array}{l}\text { Lack of knowledge (theme and } \\
\text { procedure) }(-)\end{array}$ & $\begin{array}{c}\text { (Gunasekaran et al., 2009; Haag et al., } \\
\text { 2013) }\end{array}$ \\
\hline Management efforts (-) & (Haag et al., 2013) \\
\hline Costs $(+/-)$ & $\begin{array}{c}\text { (Gunasekaran et al., 2009; Urciuoli et } \\
\text { al., 2013) }\end{array}$ \\
\hline Relative advantage $(+)$ & (Riyadh et al., 2019; Thi et al., 2014) \\
\hline IT infrastructure $(+)$ & (Gunasekaran et al., 2009; Thi et al., \\
\hline
\end{tabular}




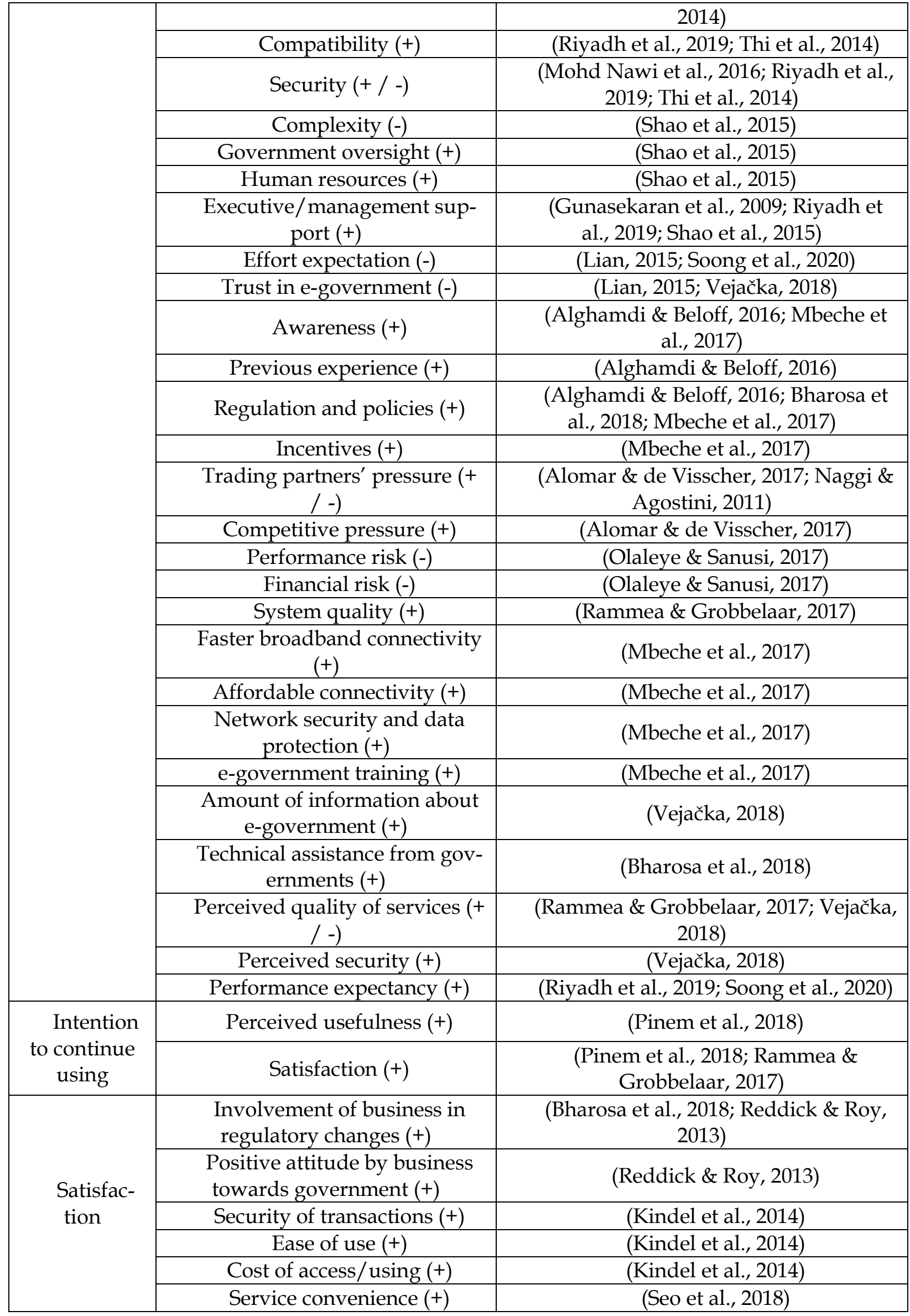




\begin{tabular}{|c|c|c|}
\hline & Performance failure (-) & (Seo et al., 2018) \\
\hline & Operational effectiveness $(+)$ & (Santa et al., 2019) \\
\hline & Quality of information (+) & (Santa et al., 2019) \\
\hline \multirow{6}{*}{$\begin{array}{l}\text { Confirma- } \\
\text { tion of sys- } \\
\text { tem adoption }\end{array}$} & $\begin{array}{l}\text { Perceived administrative bur- } \\
\text { den reduction }(+)\end{array}$ & (Arendsen et al., 2014) \\
\hline & $\begin{array}{l}\text { Successful system implementa- } \\
\text { tion }(+)\end{array}$ & (Arendsen et al., 2014) \\
\hline & Ease of use $(+)$ & (Arendsen et al., 2014) \\
\hline & Productivity $(+)$ & (Arendsen et al., 2014) \\
\hline & Usefulness (+) & (Ulman et al., 2012) \\
\hline & Time-savings $(+)$ & $\begin{array}{c}\text { (Naggi \& Agostini, 2011; Ulman et al., } \\
\text { 2012) }\end{array}$ \\
\hline \multirow{11}{*}{$\begin{array}{l}\text { Channel } \\
\text { choice }^{* *} \text { (in- } \\
\text { ternet) }\end{array}$} & Size $(+/-)$ & $\begin{array}{c}\text { (Jansen et al., 2010; van den Boer et al., } \\
\text { 2014) }\end{array}$ \\
\hline & Age $(+/-)$ & $\begin{array}{l}\text { (Jansen et al., 2010; van den Boer et al., } \\
\text { 2014) }\end{array}$ \\
\hline & Attitude $(+/-)$ & (Jansen et al., 2010) \\
\hline & Digital skills $(+)$ & (Jansen et al., 2010) \\
\hline & Source to get information $(+/-)$ & (van den Boer et al., 2012, 2016) \\
\hline & Social influence $(+)$ & (van den Boer et al., 2014) \\
\hline & Education $(+)$ & (van den Boer et al., 2014) \\
\hline & Task (specificity) (+/-) & (van den Boer et al., 2017, 2014) \\
\hline & Exact situation (+/-) & (van den Boer et al., 2016) \\
\hline & Prior experience $(+/-)$ & (van den Boer et al., 2017) \\
\hline & $\begin{array}{l}\text { Perceived expertise of the gov- } \\
\text { ernment }(+)\end{array}$ & (van den Boer et al., 2017) \\
\hline
\end{tabular}

*(+) Positive influence; (-) Obstacle $\quad$ **Factors that influence internet channel choice.

There are two moments in which the research has typically been carried out, before usage itself (intention to use, channel choice), and postadoption scenarios (satisfaction, confirmation of system adoption, intention to continue using). However, intention to use; the dependent variable, attracts more attention among the studies over usage of e-government services by businesses. Research on channel choice presents a wider view of the relationship business-to-government beyond technology, as it considers that different channels can be used in a single encounter.

The factors more explored include perceived benefits, perceived usefulness, operational performance, external pressure, social influence, organizational readiness, facilitating conditions, and ease of use. All of them measured towards intention to use. Such studies are mainly based on IT acceptance theories (Davis, 1989), widely researched in the IS domain.

From a chronological perspective, it can be noticed that in the early 2000's the most explored factors are based on theoretical models such as TAM, UTAUT, DOI, mainly related to the percep- 
tion towards technology. However, in recent years there is a growing interest towards factors much more related to public sector operation such as regulations and government oversight, also attitude towards government, trust, and risk.

With regards to research settings, amongst factors influencing usage of online self-service applications, Jansen et al. (2010) analyze channel and source choice of entrepreneurs for obtaining governmental information. Their study goes beyond respondents' preferences and analyzes situational characteristics. Although they report that the most preferred channel is the internet, businesses use different channels for different purposes in different situations. Apart from the direct contact with governments, businesses make use of intermediaries to get governmental information (van den Boer et al., 2012, 2016, 2017, 2014). Task specificity particularly influences both which source and channel will be selected (van den Boer et al., 2017, 2014). In other words, businesses use a combination of channels and sources when seeking for information.

About the usage of online self-services to transact with governments, Tung \& Rieck (2005) find a significant positive relationship between perceived benefits, external pressure as well as social influence and firms' decision to adopt e-government services. Alomar \& de Visscher (2017) find organizational readiness as a variable that affects adoption but not relative advantage. Lee et al. (2011) note that the willingness to adopt e-government increases when business users perceive high quality service provision in offline service channels. Reddick \& Roy (2013) report differences in structure, demographic characteristics and perception of government and e-government among users and non-users of e-government services. Their findings suggest a relationship between a positive perception of government and businesses' inclusion in regulatory change and satisfaction with e-government. Alghamdi \& Beloff (2016) find awareness, previous experience, perceived benefits, and regulations as relevant factors. Recently, Santa et al., (2019) found operational effectiveness and information quality as drivers of business users' satisfaction in Saudi Arabia.

Concerning usage of inter-organizational services by business users, Arendsen et al. (2006) identify differences among organizational sizes with regards to the adoption of business-togovernment data exchange systems. Organizational readiness (IT-readiness and financial readiness) also play an important role for the adoption of these e-services, thereby questioning the effectiveness of enforcement strategies (Arendsen et al., 2008). For e-invoicing services, Haag et al. (2013) report that among micro firms, lack of knowledge regarding the theme and procedure of einvoicing is an important influencing factor, while in larger firms it required huge management efforts.

Shao et al. (2015), drawing on the technology-organization-environment (TOE) framework, study factors influencing e-tax filing adoption intention by business users in China. The results showed that complexity (technological category), human resource and executive support (organizational category), and government oversight (environmental category) influence e-tax filing adoption. Finally, Bharosa et al. (2018) identified steering instruments that would influence adoption of standard business reporting systems between businesses and governmental agencies, they focus on how the government implementation strategy affects businesses adoption. 
As noted previously, there is an extensive list of factors that would influence intention to use egovernment services. We identified a total of 74 unique factors towards usage of e-government services, their influence varies depending on the context of the study (country, stakeholder, eservice, etc.) and the theoretical lenses used.

Identification of too many factors reflects the complex nature of the e-Government services. At the same time, however, it also presents a dilemma for those working in the field in terms of which factors to attend to. A mechanism-based conception based on critical realism may help alleviate this problem by identifying (a limited number of) mechanisms that underpin these factors.

\section{Mechanisms towards explanation of e-Government usage by business- es}

As noted in Section 4, existing research uncovers a plethora of factors influencing the adoption egovernment services by businesses. For a practitioner, this poses a dilemma as to which factors to focus upon. We argue that these diverse number of factors are underpinned by a rather limited number of mechanisms (Saxena \& Mcdonagh, 2017b). In this section we present our attempt to hypothesize some mechanisms that would explain e-government services usage by businesses. From a critical realist perspective, the notion of mechanism is strongly related to causality. A mechanism is a causal structure that can trigger events, it has capacities for behavior, it has causal powers (Brown et al., 2021; Bygstad \& Munkvold, 2011). Retroduction is a mode of inference in which events are explained by postulating (and identifying) mechanisms which are capable of producing them (Sayer, 2010), so far the scope of this work focuses on hypothesizing, so future work can evaluate those mechanisms to actually identify which mechanism explains the event in particular settings.

For using retroduction, we took empirical observations (in a form of factors from literature) and hypothesized a list of mechanisms that might explain usage of e-government services by businesses. Our retroduction process involved a codification from the factors found relevant for egovernment services usage by businesses, which we argue, are a manifestation of mechanisms that are activated under specific contextual settings. Figure 3 shows the categories that are the result of grouping factors and the hypothesized mechanisms, as well as the context in which they might be activated (See appendix B for details). Inner context refers to the structure, culture, capabilities of the firm, it includes perceptions and experiences; outer context includes the economic, social, political, technological, competitive and sectoral environments in which the firm is located, what the firm cannot control (Pettigrew, 1997). 
Figure 3: Retroduction process and hypothesized mechanisms

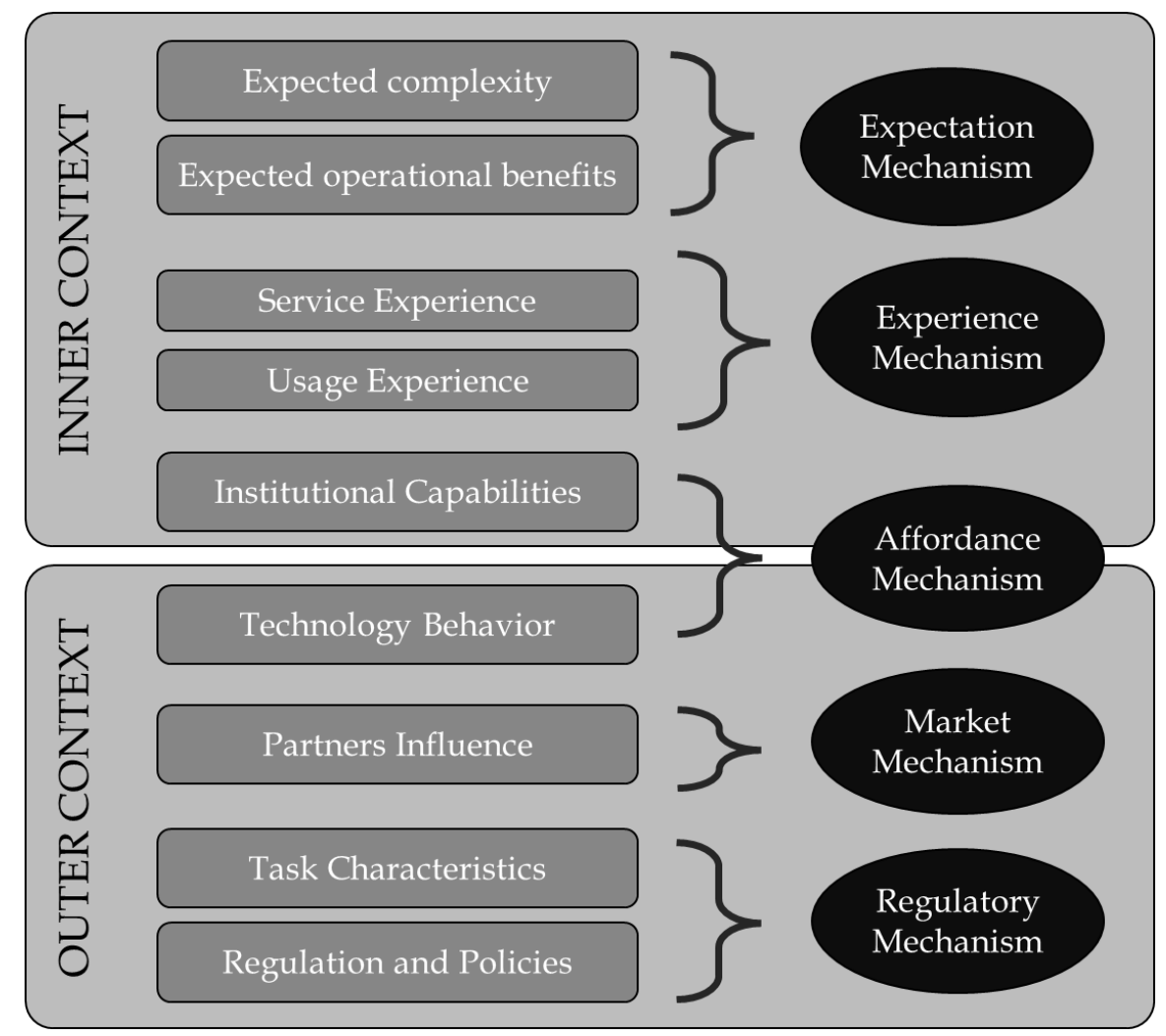

After the analysis, five mechanisms were postulated. Two mechanisms - expectation, and experience mechanism - were identified with reference to the inner context of the firm. Two mechanisms - market and regulation - were identified in relation to the outer context. One mechanism, affordance, was hypothesized to be associated both with the inner and the outer context. It related to the possibilities and constraints of firm's capabilities and technology. It can be activated from both the inner and outer context of the organization as in the context of e-government, the governmental agency shapes technology behavior. Each postulated mechanism can be described in terms of the factors they underpin. A brief description can be seen in table 12 .

Table 12. Mechanism's description

\begin{tabular}{|c|c|}
\hline Mechanism & \multicolumn{1}{c|}{ Description } \\
\hline Expectation & $\begin{array}{l}\text { According to expectation confirmation theory, expectation reflects antic- } \\
\text { ipated behavior. The role of expectation has been widely explored in the IT } \\
\text { adoption field. Users form expectations and perceptions towards complexi- } \\
\text { ty (of technology) (Davis, 1989; Lian, 2015; Shao et al., 2015) and benefits } \\
\text { (for their individual work performance or for businesses operation) (Ar- } \\
\text { endsen et al., 2006; Chwelos et al., 2001; Tung \& Rieck, 2005). Expectations } \\
\text { could influence their intention to use e-government services, whether in a } \\
\text { positive or negative way. However, the context and the interaction with } \\
\text { other mechanisms would shape the influence exerted over usage of e- } \\
\text { government services. }\end{array}$ \\
\hline Experience & $\begin{array}{l}\text { Experience with the system affects user attitudes towards the system } \\
\text { (Piehler et al., 2016). Attitude has been identified as a strong predictor of }\end{array}$ \\
\hline
\end{tabular}




\begin{tabular}{|c|c|}
\hline & $\begin{array}{l}\text { behavior towards usage of information technology (Alomar \& de Visscher, } \\
\text { 2017; Arendsen et al., 2014; Arendsen \& Van De Wijngaert, 2011), prior ex- } \\
\text { perience influences new channel choices to interact with governments (Teer- } \\
\text { ling \& Pieterson, 2011). Experience is not free of concerns (Olaleye \& Sanusi, } \\
\text { 2017), it can also contribute to the increase of trust. There are different mani- } \\
\text { festations of experience as a mechanism that explain behavior towards us- } \\
\text { age of e-government services. In fact, it is a complex mechanism that inter- } \\
\text { acts with others as experience influences expectations as well (Santa et al., } \\
\text { 2019). } \\
\text { In addition, satisfaction can only be evaluated after usage experience, it is } \\
\text { generated when expectations can be met (Pinem et al., 2018). }\end{array}$ \\
\hline Affordance & $\begin{array}{l}\text { The concept of an affordance mechanism helps explain how an object or } \\
\text { a focal technology can support or restrict a set of specific usage with refer- } \\
\text { ence to a specific user (Leonardi, 2011). In the organizational context, tech- } \\
\text { nology affordance does not refer merely to specific features of the technolo- } \\
\text { gy but also to the capability of the organization to appropriate the technolo- } \\
\text { gy artifact towards its business objective (Zammuto et al., 2007). } \\
\text { Certain affordances emerge from the designer of the technology who } \\
\text { have some intended use in their mind. E-government services are devel- } \\
\text { oped by governmental agencies, the web design, their performance, their } \\
\text { failures, security, among other technical aspects, can trigger events, such as } \\
\text { decisions users make towards usage of e-services. However, not all af- } \\
\text { fordances are realized due to firms' specific context. Firm's resources and } \\
\text { capabilities relate to technical and organizational infrastructure that allow } \\
\text { or constrain technology use. In this context, resources and capabilities in- } \\
\text { clude the availability of IT infrastructure, knowledge (IT and task), financial } \\
\text { resources. Last but not the least, realization of affordance also relates to the } \\
\text { processes by which the firm gets ready for the implementation and use of e- } \\
\text { government services. }\end{array}$ \\
\hline Market & $\begin{array}{l}\text { Market is a mechanism that has been identified in different contexts, } \\
\text { mainly represented in the influence that partners exert on the firm behavior. } \\
\text { Whether due to competitive pressure (to maintain their own competitive } \\
\text { position), social influence (if the behavior is viewed more favorably by the } \\
\text { public or by other organizations), or trading partners pressure (pressure ex- } \\
\text { erted by associates) (Alomar \& de Visscher, 2017; Chwelos et al., 2001; Tung } \\
\text { \& Rieck, 2005). Firms can take an initiative in adopting e-government ser- } \\
\text { vices when market mechanism is activated. } \\
\text { The structure of the market, the relationship of a firm with its competi- } \\
\text { tors and the relationship of a firm with suppliers and customers can shape } \\
\text { firm's behavior and decisions towards technology. }\end{array}$ \\
\hline Regulation & $\begin{array}{l}\text { Regulation has the power to seduce or enforce (Arendsen et al., 2008). } \\
\text { Usage of e-government services can be voluntary, or mandatory based on } \\
\text { normativity. Government has the power to restrict decisions that firms can } \\
\text { make based on the regulatory framework applicable. They provide the rules } \\
\text { that enterprises must follow; it includes decisions towards usage of technol- } \\
\text { ogy. } \\
\text { Regulation determines the rules for the tasks businesses must perform } \\
\text { (administrative burden), but also can limit the channel choices to perform } \\
\text { those tasks. Task-technology fit (TTF) theory holds that IT is more likely to }\end{array}$ \\
\hline
\end{tabular}


be used if IT matches the tasks that the user must perform (Goodhue \& Thompson, 1995). Businesses are responsible for different tasks (administrative burden) as part of their operation, the characteristics of those tasks influence which channels are used.

The philosophy of critical realism acknowledges that mechanisms not necessarily work in isolation, they may interact with each other to generate causal effects. As Sayer (2010) observes, the causal powers of mechanisms do not operate in single objects or individuals but in the social relations and structures which they form. Adoption of e-government services can be seen as a process, rather than a single decision, thus diverse mechanisms are activated at different stages such as before, during and even after usage of e-services.

Within internal context, Expectation and experience mechanisms seem to interact with each other throughout usage event, individuals hold expectations that are confirmed or disconfirmed towards experiences and then experience will shape new expectations. Affordance mechanisms also play a role, since both, expectation and experience mechanisms are activated based on the possibilities of technology and the capabilities of the users.

Within the external context, regulation and market mechanism interact towards usage of eservices, however, affordance mechanism plays a part here too. Although actors in the market (such as suppliers, customers, competitors) or government agencies (through regulation) have the power to influence a firm's behavior, institutional infrastructure (affordances) determines how the firm can respond to those influences.

Hence, affordance mechanism can be activated in the inner context of the organization (institutional capabilities), but also in the outer context of the organization (technology provided by the government). However, irrespective of the location of initial conceptualization, affordance realization is always a product of designers' (e-government service provider) intentions and the capability of the users (businesses using the services) (Bansal \& Shukla, 2021; Brown et al., 2021; Faraj \& Azad, 2012).

\section{Conclusions and future work}

This literature review analyzed research on the field of a B2G technology-mediated relationship with a particular focus on the businesses' perspective, which means businesses involvement as a source of information in the empirical studies. We presented an overview of 38 empirical papers studying the usage of e-Government services by businesses. They were selected following Webster \& Watson (2002); classified based on the research onion model (Saunders et al., 2019) and additional categories; and main findings were analyzed in order to hypothesize possible mechanisms that would explain the usage of e-services by enterprises.

We identify 74 unique factors that would influence e-government usage. These factors vary among different contexts (countries, services, businesses sizes). Based on a retroductive analysis of these factors, we postulate 5 mechanisms that might be involved in the phenomenon of usage of e- 
government. These mechanisms interact with each other and have the power to influence adoption or non-adoption of e-services. We argue that the factors from which the mechanisms have emerged are a manifestation of them.

There are limitations of our study, our search equation did not include names of specific eservices such as e-invoicing, e-taxation, e-procurement that are related to B2G relationship and in which more empirical work could have been found. Nevertheless, as our search terms were wider in nature, a good overview of the field was obtained. A second limitation of the study stems from the secondary nature of the evidence. Empirical studies are needed to validate the existence and the operation of hypothesized mechanisms based on primary data.

Based on the findings in this paper, we present our suggestions for future research based on methodological aspects, and themes. Regarding methodological aspects, future research may include more qualitative studies, which can help to understand the phenomenon of usage of egovernment services in depth. Qualitative studies can be conducted to study the enactment and interaction of mechanisms. On the other hand, the incorporation of longitudinal studies, (as most existing research is cross-sectional in nature) might help to understand usage of e-government as a process, beyond a decision to adopt, thus helping the practitioners to devise strategies towards the uptake of e-services.

With respect to actors, although usage of e-services commonly states a set of benefits for businesses, we suggest that research can help uncover the actual benefits of the electronic mediated relationship between businesses and governments. We need to move beyond the perceived benefits and investigate actual benefits to the business, since the realization of benefits would keep businesses engaged in using electronic services. In addition, intermediaries (formal and social), play a role in the relationship between businesses and governments (Arendsen et al., 2006; Arendsen \& Ter Hedde, 2009; Bharosa et al., 2018; van den Boer et al., 2012) in the context of egovernment research, thus deserving more attention from the researchers.

Finally, this study contributes to theory building by using critical realism, as opposed to the widely used positivist approach. Thus, this study provides an alternative explanation for the usage of e-government services based on mechanisms.

\section{References}

Alghamdi, S., \& Beloff, N. (2016). Innovative Framework for e-Government adoption in Saudi Arabia: A Study from the business sector perspective. International Journal of Advanced Computer Science and Applications, 7(1). https://doi.org/10.14569/ijacsa.2016.070189

Alomar, M. A., \& de Visscher, C. (2017). Which Factors Can Affect e-Public Procurement Adoption by Private Firms? The Case of Belgium. Electronic Journal of E-Government, 15(2), 103-115.

Arendsen, R., Peters, O., ter Hedde, M., \& van Dijk, J. (2014). Does e-government reduce the administrative burden of businesses? An assessment of business-to-government systems usage in the Netherlands. Government Information Quarterly, 31(1), 160-169. https:// doi.org/10.1016/j.giq.2013.09.002 
Arendsen, R., \& Ter Hedde, M. J. (2009). On the origin of intermediary e-government services. Lecture Notes in Computer Science (Including Subseries Lecture Notes in Artificial Intelligence and Lecture Notes in Bioinformatics), 5693 LNCS, 270-281. https://doi.org/10.1007/978-3-642-03516-6_23

Arendsen, R., \& Van De Wijngaert, L. (2011). Government as a launching customer for elnvoicing. Lecture Notes in Computer Science (Including Subseries Lecture Notes in Artificial Intelligence and Lecture Notes in Bioinformatics), 6846 LNCS, 122-133. https:/ / doi.org/10.1007/978-3-642-22878-0_11

Arendsen, R., \& van Engers, T. M. (2004). Reduction of the Administrative Burden: An e-Government Perspective. In R. Traunmüller (Ed.), Electronic Government: Proceedings of the 3rd IFIP WG 8.5 International Conference, EGOV $2004 \quad$ (Vol. 3183, pp. 200-206). http://www.springerlink.com/openurl.asp?genre=article\&id=BHJ6V5EJU1DW8P1D

Arendsen, R., Van Engers, T. M., \& Schurink, W. (2008). Adoption of high impact governmental eServices: Seduce or enforce? Lecture Notes in Computer Science, 5184, 73-84. https:// doi.org/10.1007/978-3-54085204-9

Arendsen, R., Van Engers, T. M., \& Velde, R. (2006). An Empirical Study on Business-to- Government Data Exchange Strategies to Reduce the Administrative Costs for Businesses. In R. Suomi, R. Cabral, J. F. Hampe, A. Heikkilä, J. Järveläinen, \& E. Koskivaara (Eds.), Project E-Society: Building Bricks. IFIP International Federation for Information Processing (pp. 311-323). Springer, Boston, MA.

Bansal, V., \& Shukla, S. (2021). Exploring Big Data Analytics Adoption using Affordance Theory. Proceedings of the 23rd International Conference on Enterprise Information System, 2: ICEIS, 131-138. https://doi.org/10.5220/0010509801310138

Bharosa, N., Hietbrink, F., Mosterd, L., \& Van Oosterhout, R. (2018). Steering the adoption of Standard Business Reporting for cross domain information exchange. ACM International Conference Proceeding Series. https://doi.org/10.1145/3209281.3209325

Brown, S., Saxena, D., \& Wall, P. J. (2021). The role of information and communications technology in refugee entrepreneurship: A critical realist case study. The Electronic Journal of Information Systems in Developing Countries, December 2020, 1-20. https://doi.org/10.1002/isd2.12195

Bygstad, B., \& Munkvold, B. E. (2011). In search of mechanisms. Conducting a critical realist data analysis. International Conference on Information Systems 2011, ICIS 2011, 3, 1978-1992.

Chwelos, P., Benbasat, I., \& Dexter, A. S. (2001). Research Report: Empirical Test of an EDI Adoption Model. Information Systems Research, 12(3), 304-321. https://doi.org/10.1287/isre.12.3.304.9708

Davis, F. D. (1989). Perceived Usefulness, Perceived Ease Of Use, and User Acceptance of Information Technology. MIS Quarterly, 13(3), 319-339. https:// doi.org/10.2307/249008

DeLone, W. H., \& McLean, E. R. (1992). Information systems success: the quest for the dependent variable. Information Systems Research, 3(1), 60-95.

DeLone, W. H., \& McLean, E. R. (2003). The DeLone and McLean Model of Information Systems Success: A Ten-Year Update. Journal of Management Information Systems, 19(4), 9-30. https://doi.org/10.1080/07421222.2003.11045748

Distel, B., \& Ogonek, N. (2016). To Adopt or not to Adopt: A Literature Review on Barriers to Citizen's Adoption of e-Government Services. Twenty-Fourth European Conference on Information Systems (ECIS). 
Dwivedi, Y., Rana, N. P., Janssen, M., Lal, B., Williams, M. D., \& Clement, M. (2017). An empirical validation of a unified model of electronic government adoption (UMEGA). Government Information Quarterly, 34(2), 211-230. https://doi.org/10.1016/j.giq.2017.03.001

Eggers, W. D., \& Bellman, J. (2015). The journey to government's digital transformation - A Deloitte Digital global survey.

Faraj, S., \& Azad, B. (2012). The Materiality of Technology: An Affordance Perspective. In P. M. Leonardi, B. A. Nardi, \& J. Kallinikos (Eds.), Material- ity and organizing: Social interaction in a technological world (pp. 237-258). Oxford University Press. https://doi.org/10.1093/acprof

Goodhue, D., \& Thompson, R. (1995). Task-Technology Fit and Individual Performance. MIS Quarterly, 19(2), 213-236. https://doi.org/10.2307/249689

Gunasekaran, A., McGaughey, R. E., Ngai, E. W. T., \& Rai, B. K. (2009). E-Procurement adoption in the Southcoast SMEs. International Journal of Production Economics, 122(1), 161-175. https://doi.org/10.1016/j.ijpe.2009.05.013

Haag, S., Born, F., Kreuzer, S., \& Bernius, S. (2013). Organizational resistance to e-invoicing-results from an empirical investigation among smes. Lecture Notes in Computer Science (Including Subseries Lecture Notes in Artificial Intelligence and Lecture Notes in Bioinformatics), 8074 LNCS, 286-297. https://doi.org/10.1007/978-3-642-40358-3-24

Hofmann, S., Räckers, M., \& Becker, J. (2012). Identifying factors of e-Government acceptance - A literature review. International Conference on Information Systems.

Hung, S.-Y., Chang, C.-M., Chen, K., Tang, K.-Z., \& Chou, C.-H. (2012). Buyer acceptance of g2b egovernment services. ACM SIGMIS Database: The DATABASE for Advances in Information Systems, 42(4), 81-97. https://doi.org/10.1145/2096140.2096146

Jansen, J., Wijngaert, L. van de, \& Pieterson, W. (2010). Channel choice and source choice of entrepreneurs in a public organizational context: the dutch case. In M. A. Wimmer, J.-L. Chappelet, M. Janssen, \& H. J. Scholl (Eds.), Electronic Government: Proceedings of the 9th IFIP WG 8.5 International Conference, EGOV 2010 (Vol. 6228, pp. 144-155).

Kassim, E. S., \& Hussin, H. (2010). An Integrative Approach to Inter-Organizational System Implementation and Evaluation: the Case of Government-to -Business. Communications of the IBIMA, 2010. https://doi.org/10.5171/2010.694811

Kindel, K., Ritso, V., \& Venesaar, U. (2014). Business satisfaction with E-government services in Baltic sea region. Frontiers in Artificial Intelligence and Applications, Databases, 217-230. https://doi.org/10.3233/978-1-61499-458-9-217

Lampoltshammer, T. J., Zhu, Q., \& Parycek, P. (2019). Affective effect: Issue engagement on a youth eparticipation platform. EJournal of EDemocracy and Open Government, 11(1), 37-58. https://doi.org/10.29379/jedem.v11i1.558

Lee, J., Kim, H. J., \& Ahn, M. J. (2011). The willingness of e-Government service adoption by business users: The role of offline service quality and trust in technology. Government Information Quarterly, 28(2), 222-230. http://www.sciencedirect.com/science/article/pii/S0740624X10001346 
Leonardi, Paul M. (2011). When flexible routines meet flexible technologies: Affordance, constraint, and the imbrication of human and material agencies. MIS Quarterly: Management Information Systems, 35(1), 147-167. https://doi.org/10.2307/23043493

Lian, J. W. (2015). Critical factors for cloud based e-invoice service adoption in Taiwan: An empirical study. International Journal of Information Management, 35(1), 98-109. https://doi.org/10.1016/j.ijinfomgt.2014.10.005

MacDonald, C. (2017). Digital transformation and the public sector. Organisation for Eco-nomic Cooperation and Development. http://www.oecd.org/gov/digital-government/digital-transformation-andthe-public-sector.htm

Madsen, C. Ø., \& Kræmmergaard, P. (2015). Channel Choice: A Literature review. EGOV 2015. Lecture Notes in Computer Science. https:/ / doi.org/10.1007/978-3-319-22479-4

Madsen, C. Ø., \& Kræmmergaard, P. (2016). Warm Experts in the Age of Mandatory e-Government: Interaction Among Danish Single Parents Regarding Online Application for Public Benefits. Electronic Journal of E-Government, 14(1), 87-98.

Mbeche, E. M., Okeyo, G., \& Kimwele, M. (2017). Critical Success Factors Influencing Adoption and Use of E-government Services by SMEs in the Manufacturing Sector in Kenya. International Advanced Research Journal in Science, Engineering and Technology, 4(6), 16-24. https://doi.org/10.17148/iarjset.2017.4604

Mergel, I., Kattel, R., Lember, V., \& McBride, K. (2018). Citizen-oriented digital transformation in the public sector. Proceedings of the 19th Annual International Conference on Digi-Tal Government Research: Governance in the Data Age, 1-3.

Mohd Nawi, M. N., Roslan, S., Salleh, N. A., Zulhumadi, F., \& Harun, A. N. (2016). The benefits and challenges of E-procurement implementation: A case study of Malaysian company. International Journal of Economics and Financial Issues, 6(7Special Issue), 329-332.

Naggi, R., \& Agostini, P. L. (2011). Inter-organizational e-Services from a SME Perspective: A Case Study on e-Invoicing. Information Technology and Innovation Trends in Organizations. https://doi.org/10.1007/978-3-7908-2632-6

Nguyen, H. T., Dang, T. V., Van Nguyen, V., \& Nguyen, T. T. (2020). Determinants of e-government service adoption: an empirical study for business registration in Southeast Vietnam. Journal of Asian Public Policy, 1-16. https:/ / doi.org/10.1080/17516234.2020.1805396

Nunes, S., Martins, J., Branco, F., Goncalves, R., \& Au-Yong-Oliveira, M. (2017). An initial approach to egovernment acceptance and use: A literature analysis of e-Government acceptance determinants. Iberian Conference on Information Systems and Technologies, CISTI. https://doi.org/10.23919/CISTI.2017.7976044

Olaleye, S. A., \& Sanusi, I. T. (2017). Companies Resistance and Intention to use Electronic Invoicing in Nigeria. 2017 14th IEEE India Council International Conference (INDICON). https://doi.org/10.1109/INDICON.2017.8487863

Pettigrew, A. M. (1997). What is a processual analysis? Pergamon, 13(4), 337-348.

Piehler, R., Wirtz, B. W., \& Daiser, P. (2016). An Analysis of Continuity Intentions of eGovernment Portal Users. Public Management Review, 18(2), 163-198. https://doi.org/10.1080/14719037.2014.965270 
Pinem, A. A., Immanuella, I. M., Hidayanto, A. N., Phusavat, K., \& Meyliana. (2018). Trust and its impact towards continuance of use in government-to-business online service. Transforming Government: People, Process and Policy, 12(3-4), 265-285. https:/ / doi.org/10.1108/TG-02-2018-0008

Rammea, L., \& Grobbelaar, S. S. (2017). The Evaluation of e-Government Implementation. IEEE Africon 2017 Proceedings.

Reddick, C. G., \& Roy, J. (2013). Business perceptions and satisfaction with e-government: Findings from a Canadian survey. Government Information Quarterly, 30(1), 1-9. https://doi.org/10.1016/j.giq.2012.06.009

Riyadh, H. A., Alfaiza, S. A., \& Sultan, A. A. (2019). The effects of technology, organisational, behavioural factors towards utilization of egovernment adoption model by moderating cultural factors. Journal of Theoretical and Applied Information Technology, 97(8), 2142-2165.

Rogers, E. M. (1995). Diffusion of innovations (3rd Editio). Free Press.

Ruvalcaba-Gomez, E. A. (2019). Open government and citizen participation: Perceptions between civil society organizations and government. EJournal of EDemocracy and Open Government, 11(2), 1-13. https://doi.org/10.29379/jedem.v11i2.539

Sambasivan, M., Wemyss, G. P., \& Rose, R. C. (2010). User acceptance of a G2B system: A case of electronic procurement system in Malaysia. Internet Research, 20(2), 169-187. https://doi.org/10.1108/10662241011032236

Santa, R., MacDonald, J. B., \& Ferrer, M. (2019). The role of trust in e-Government effectiveness, operational effectiveness and user satisfaction: Lessons from Saudi Arabia in e-G2B. Government Information Quarterly, 36(1), 39-50. https://doi.org/10.1016/j.giq.2018.10.007

Saunders, M. N. K., Lewis, P., \& Thornhill, A. (2019). Research Methods for Business Students (8th ed.). Pearson.

Saxena, D. (2019). The Search for Mechanisms in Business Research : Reflections on Retroductive Analysis in a Multilevel Critical Realist Case Study. Electronic Journal of Business Research Methods, 17(1), 17-27.

Saxena, D., \& Mcdonagh, J. (2017a). A systematic literature review of the enterprise systems research in leading IS journals(2000-2015). Proceedings of the Twelfth Midwest Association for Information Systems

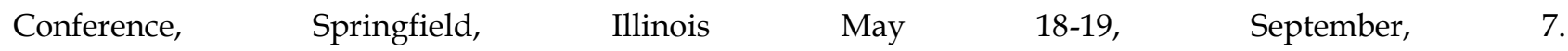
https://www.researchgate.net/publication/318116965

Saxena, D., \& Mcdonagh, J. (2017b). Yet Another "List" of Critical Success "Factors" for Enterprise Systems: Review of Empirical Evidence and Suggested Research Directions. UKAIS: 22nd Annual Conference: Ubiquitous Information Systems: Surviving and Thriving in a Connected Society. https://aisel.aisnet.org/ukais2017/66

Sayer, A. (2010). Method in social science: A realist approach (2nd ed.). Routledge.

Scholta, H., Halsbenning, S., Distel, B., \& Becker, J. (2020). Walking a Mile in Their Shoes - A Citizen Journey to Explore Public Service Delivery from the Citizen Perspective. Electronic Government: 19th IFIP WG 8.5 International Conference, Proceedings, 164-178. 
Seo, D. B., Tan, C. W., \& Warman, G. (2018). Vendor satisfaction of E-government procurement systems in developing countries: an empirical research in Indonesia. Information Technology for Development, 24(3), 554-581. https://doi.org/10.1080/02681102.2018.1454878

Shao, B., Luo, X. (Robert), \& Liao, Q. (2015). Factors influencing e-tax filing adoption intention by business users in China. Electronic Government, an International Journal, 11(4), 283-305. https://doi.org/10.1504/EG.2015.071408

Soong, K. K., Ahmed, E. M., \& Tan, K. S. (2020). Factors influencing Malaysian small and medium enterprises adoption of electronic government procurement. Journal of Public Procurement, 20(1), 38-61. https://doi.org/10.1108/JOPP-09-2019-0066

Teerling, M. L., \& Pieterson, W. (2011). How to improve e-government use: An empirical examination of multichannel marketing instruments. Information Polity: The International Journal of Government \& Democracy in the Information Age, 16(2), 171-187. https:/ / doi.org/10.3233/IP-2011-0213

Thi, L., Lim, H., \& Al-Zoubi, M. I. (2014). Estimating influence of TOE factors on E-Government usage: Evidence of Jordanian companies. International Journal of Business and Society, 15(3), 413-436.

Tung, L. L., \& Rieck, O. (2005). Adoption of electronic government services among business organizations in Singapore. The Journal of Strategic Information Systems, 14(4), 417-440. https://doi.org/10.1016/j.jsis.2005.06.001

Ulman, M., Jarolímek, J., Vasilenko, A., \& Kánská, E. (2012). The evaluation of use and quality of public eservices among enterprises. Agris On-Line Papers in Economics and Informatics, 4(2), 61-72.

Urciuoli, L., Hintsa, J., \& Ahokas, J. (2013). Drivers and barriers affecting usage of e-Customs - A global survey with customs administrations using multivariate analysis techniques. Government Information Quarterly, 30(4), 473-485. https://doi.org/10.1016/j.giq.2013.06.001

van den Boer, Y., Arendsen, R., \& Pieterson, W. (2016). In search of information: Investigating source and channel choices in business-to-government service interactions. Government Information Quarterly, 33(1), 40-52. https://doi.org/10.1016/j.giq.2015.11.010

van den Boer, Y., Pieterson, W., \& Arendsen, R. (2011). Channel Choice in the G2B Context: Towards a Research Agenda. In IFIP International Federation for Information Processing (pp. 14-27). https://doi.org/10.1007/978-3-642-27260-8_2

van den Boer, Y., Pieterson, W., Arendsen, R., \& De Groot, M. (2014). Source and Channel Choices in Business-to-Government Service Interactions: A Vignette Study. IFIP International Federation for Information Processing 2014, 8653 LNCS, 120-132. https:// doi.org/10.1007/978-3-662-44426-9

van den Boer, Y., Pieterson, W., Arendsen, R., \& van Dijk, J. (2017). Towards a model of source and channel choices in business-to-government service interactions: A structural equation modeling approach. Government Information Quarterly, 34(3), 434-456. https://doi.org/10.1016/j.giq.2017.07.002

van den Boer, Y., van de Wijngaert, L., Pieterson, W., \& Arendsen, R. (2012). On the interaction of source and channel choice in the government-to- business context. Lecture Notes in Computer Science (Including Subseries Lecture Notes in Artificial Intelligence and Lecture Notes in Bioinformatics), 7443 LNCS, 27-39. https://doi.org/10.1007/978-3-642-33489-4_3 
Vejačka, M. (2018). Acceptance of e-government services by business users: The case of Slovakia. Journal of Applied Economic Sciences, 13(5), 1409-1417.

Webster, J., \& Watson, R. T. (2002). Analyzing the Past to Prepare for the Future: Writing a Literature Review. MIS Quarterly, 26(2), xiii-xxiii. https://doi.org/10.1.1.104.6570

Zammuto, R. F., Griffith, T. L., Majchrzak, A., Dougherty, D. J., \& Faraj, S. (2007). Information technology and the changing fabric of organization. Organization Science, 18(5), 749-762. https://doi.org/10.1287/orsc.1070.0307

\begin{abstract}
About the Author
Martha Correa-Ospina

Martha Correa is a PhD Candidate at the Department of Systems and Industrial Engineering, Faculty of Engineering, Universidad Nacional de Colombia-Bogotá. Her doctoral research is related to the uptake of e-government services by businesses (focused on micro businesses). Her research interests focus on the uptake, assessment, and value of information technology in organizations (particularly, public sector).

\section{Deepak Saxena}

Deepak Saxena is an Assistant Professor at the Department of Management, Birla Institute of Technology and Science Pilani. He holds a PhD from Trinity College Dublin (Ireland). His areas of interest include digital transformation, public services, and critical realism. His works have appeared in journals such as Australasian Journal of Information Systems, Electronic Journal of IS Evaluation, Electronic Journal of eGovernment, Irish Journal of Management, Journal of Information Science, and Journal of Information Technology Teaching Cases.
\end{abstract}

\title{
Beatriz Helena Díaz Pinzón
}

Beatriz Díaz is an Associate Professor at the Business School, Faculty of Economic Sciences, Universidad Nacional de Colombia-Bogotá. She is a Systems Engineer from the same University, and she holds a Master of management of Information Systems and Organizations, and a PhD in Management Sciences from the University of Grenoble (France). She is the director of the GISTIC research group. Her research areas include management information systems and education. 
Appendix A: Pool of papers

\begin{tabular}{|c|c|c|}
\hline Sample (Business size) & $\begin{array}{l}\text { Country of data collec- } \\
\text { tion }\end{array}$ & $\begin{array}{l}\text { Reference to the paper (refer- } \\
\text { ences section) }\end{array}$ \\
\hline Entrepreneurs & The Netherlands & (Jansen et al., 2010) \\
\hline $\begin{array}{l}\text { Micro, Small and Medi- } \\
\text { um }\end{array}$ & Germany & (Haag et al., 2013) \\
\hline \multirow{10}{*}{ Small and Medium } & The Netherlands & (Arendsen et al., 2006) \\
\hline & The United States & (Gunasekaran et al., 2009) \\
\hline & The Netherlands & (van den Boer et al., 2012) \\
\hline & The Netherlands & (van den Boer et al., 2014) \\
\hline & Multiple locations & (Kindel et al., 2014) \\
\hline & The Netherlands & (van den Boer et al., 2016) \\
\hline & The Netherlands & (van den Boer et al., 2017) \\
\hline & Kenya & (Mbeche et al., 2017) \\
\hline & Vietnam & (Nguyen et al., 2020) \\
\hline & Malaysia & $\begin{array}{l}\text { (Kassim \& Hussin, 2010; Soong et } \\
\text { al., 2020) }\end{array}$ \\
\hline Medium & Italy & (Naggi \& Agostini, 2011) \\
\hline \multirow{3}{*}{ Large } & Singapore & (Tung \& Rieck, 2005) \\
\hline & The Netherlands & (Arendsen et al., 2008) \\
\hline & The Netherlands & $\begin{array}{l}\text { (Arendsen \& Van De Wijngaert, } \\
\text { 2011) }\end{array}$ \\
\hline \multirow{21}{*}{ All sizes / not specified } & Malaysia & (Sambasivan et al., 2010) \\
\hline & Korea & (Lee et al., 2011) \\
\hline & Taiwan & (Hung et al., 2012) \\
\hline & Czech Republic & (Ulman et al., 2012) \\
\hline & Canada & (Reddick \& Roy, 2013) \\
\hline & Multiple locations & (Urciuoli et al., 2013) \\
\hline & The Netherlands & (Arendsen et al., 2014) \\
\hline & Jordan & (Thi et al., 2014) \\
\hline & China & (Shao et al., 2015) \\
\hline & Taiwan & (Lian, 2015) \\
\hline & Saudi Arabia & (Alghamdi \& Beloff, 2016) \\
\hline & Malaysia & (Mohd Nawi et al., 2016) \\
\hline & Belgium & (Alomar \& de Visscher, 2017) \\
\hline & Nigeria & (Olaleye \& Sanusi, 2017) \\
\hline & Lesotho & (Rammea \& Grobbelaar, 2017) \\
\hline & Indonesia & (Seo et al., 2018) \\
\hline & Indonesia & (Pinem et al., 2018) \\
\hline & Slovakia & (Vejačka, 2018) \\
\hline & The Netherlands & (Bharosa et al., 2018) \\
\hline & Iraq & (Riyadh et al., 2019) \\
\hline & Saudi Arabia & (Santa et al., 2019) \\
\hline
\end{tabular}


Appendix B: Retroduction process

\begin{tabular}{|c|c|c|c|}
\hline Factor & Category & $\begin{array}{l}\text { Hypothesized } \\
\text { mechanism }\end{array}$ & Context \\
\hline Effort expectation & \multirow{5}{*}{$\begin{array}{l}\text { Expected complexi- } \\
\text { ty }\end{array}$} & \multirow{15}{*}{$\begin{array}{l}\text { Expectation } \\
\text { mechanism }\end{array}$} & \multirow{38}{*}{$\begin{array}{l}\text { Inner } \\
\text { context }\end{array}$} \\
\hline Complexity & & & \\
\hline Ease of use & & & \\
\hline Age & & & \\
\hline Fear of change & & & \\
\hline Service convenience & \multirow{10}{*}{$\begin{array}{l}\text { Expected opera- } \\
\text { tional benefits }\end{array}$} & & \\
\hline Operational performance & & & \\
\hline Operational effectiveness & & & \\
\hline Perceived usefulness & & & \\
\hline Perceived benefits & & & \\
\hline Relative advantage & & & \\
\hline Cost of access/using & & & \\
\hline $\begin{array}{l}\text { Perceived administrative bur- } \\
\text { den reduction }\end{array}$ & & & \\
\hline Cost-savings & & & \\
\hline Performance expectancy & & & \\
\hline $\begin{array}{l}\text { Perception of high-quality of- } \\
\text { fline service provision }\end{array}$ & \multirow{6}{*}{ Service experience } & \multirow{18}{*}{$\begin{array}{l}\text { Experience } \\
\text { mechanism }\end{array}$} & \\
\hline Perceived quality of services & & & \\
\hline Service convenience & & & \\
\hline Previous experience & & & \\
\hline Prior experience & & & \\
\hline Satisfaction & & & \\
\hline Productivity & \multirow{12}{*}{ Usage experience } & & \\
\hline $\begin{array}{l}\text { Successful system implementa- } \\
\text { tion }\end{array}$ & & & \\
\hline Ease of use & & & \\
\hline $\begin{array}{l}\text { Perceived administrative bur- } \\
\text { den reduction }\end{array}$ & & & \\
\hline Trust in e-government & & & \\
\hline $\begin{array}{l}\text { Perceived expertise of the gov- } \\
\text { ernment }\end{array}$ & & & \\
\hline $\begin{array}{l}\text { Positive attitude towards gov- } \\
\text { ernment }\end{array}$ & & & \\
\hline Performance risk & & & \\
\hline Perceived security & & & \\
\hline Financial risk & & & \\
\hline Time-savings & & & \\
\hline Perceived Risk & & & \\
\hline Organizational readiness & \multirow{5}{*}{$\begin{array}{l}\text { Institutional capa- } \\
\text { bilities }\end{array}$} & \multirow{5}{*}{$\begin{array}{l}\text { Affordance } \\
\text { mechanism }\end{array}$} & \\
\hline Digital skills & & & \\
\hline Human resources & & & \\
\hline IT infrastructure & & & \\
\hline Self-efficacy & & & \\
\hline
\end{tabular}




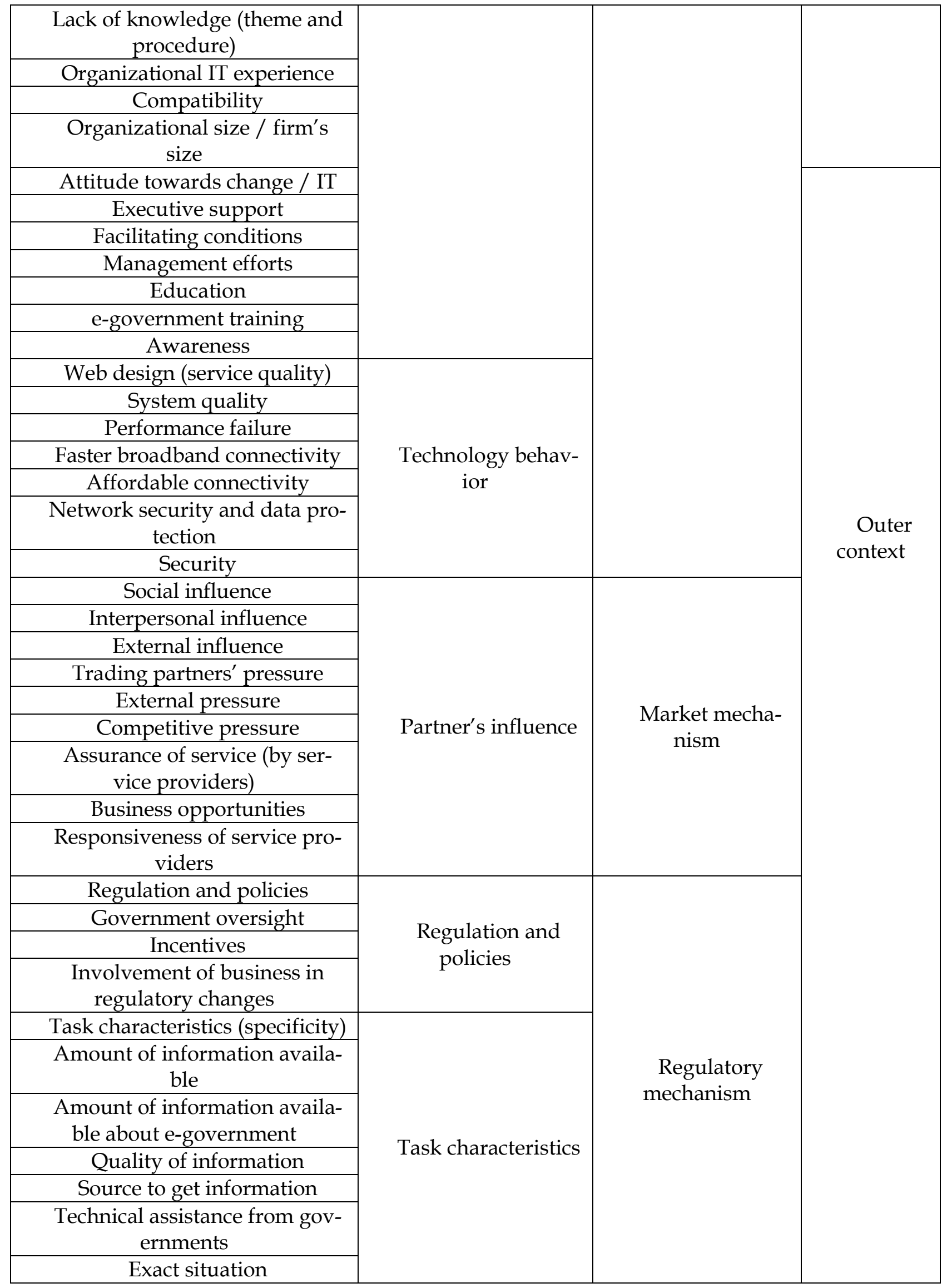

